\title{
An Email Survey of Physician and Licensed Midwife Vaccination Practices in Washington State in 2011
}

\author{
Marisa Anne D'Angeli ${ }^{1}$ and Linda O'Neal Eckert ${ }^{2}$ \\ ${ }^{1}$ Communicable Disease Epidemiology, Washington State Department of Health, 1610 NE 150th Street, Shoreline, WA 98155, USA \\ ${ }^{2}$ Department of Obstetrics and Gynecology, University of Washington, 325 9th Avenue, P.O. Box 359865, Seattle, WA 98104, USA \\ Correspondence should be addressed to Marisa Anne D’Angeli; marisa.dangeli@doh.wa.gov
}

Received 5 September 2013; Accepted 10 October 2013; Published 11 February 2014

Academic Editors: B. Best and I. Bonmarin

Copyright ( 2014 M. A. D’Angeli and L. O. Eckert. This is an open access article distributed under the Creative Commons Attribution License, which permits unrestricted use, distribution, and reproduction in any medium, provided the original work is properly cited.

\begin{abstract}
Despite proven benefit to mothers and infants, influenza and Tdap vaccination for pregnant women remain suboptimal. We conducted an email survey for physicians (MDs) and licensed midwives (LMs) in Washington to assess vaccination practices. The Washington State Department of Health and University of Washington, Department of Obstetrics and Gynecology, created an electronic survey and sent it to 644 providers. We used chi-square statistic for comparisons, with Fisher's exact test for cell size smaller than 5. We received responses from 121 (19\%), 106 of whom provided prenatal or obstetric care: 81 MDs and 25 LMs. MDs were more likely than LMs to ask whether pregnant patients are current on vaccinations for influenza and pertussis (96\% versus $56 \%$ and $84 \%$ versus $40 \%$, resp., $P<0.001)$ and to recommend influenza and pertussis vaccine during pregnancy (100\% versus $20 \%$ and $86 \%$ versus $24 \%$, resp., $P<0.001$ ). Significantly more MDs received influenza vaccine in the most recent season than did LMs (99\% versus $20 \%, P<0.001)$. In this study, LMs were less likely to inquire about immunization status, recommend influenza and pertussis vaccines, or be vaccinated against influenza than MDs. Enhancing educational communication with LMs deserves further study and may provide an opportunity to improve immunization rates in pregnant women.
\end{abstract}

\section{Introduction}

Both influenza and pertussis pose significant risks to infants. Pregnant and postpartum women and their infants are at increased risk of complications from influenza [1-4]. Multiple studies have shown that influenza immunization of women before or during pregnancy can protect both mothers and infants from influenza [5-8]. The Advisory Committee on Immunization Practices (ACIP) has recommended influenza vaccine for pregnant women in the second or the third trimester since 1997 [9] and for those in any trimester since 2004 [10]. Despite these recommendations, the proportion of pregnant women vaccinated for influenza was $15 \%$ before 2009. After the focused attention on influenza in the 2009 H1N1 influenza pandemic, the influenza immunization rate among pregnant women increased to $51 \%$ in 2010 [11] but remains suboptimal.

Infants younger than 1 year are at high risk of severe illness and death from pertussis $[12,13]$ and a majority of infant pertussis cases are infected by a family member [14, 15]. Tdap vaccination of mothers before delivery may prevent maternal infection, thus protecting the infant from exposure, and provides transplacental maternal pertussis antibodies to the infant, thereby providing some protection [16-19]. In 2012, the ACIP recommended that women receive a dose of Tdap with each pregnancy and the optimal timing for this dose is between 27 and 36 weeks of gestation [20]. The proportion of pregnant women vaccinated for pertussis is unknown, but for the general US population it is approximately $8 \%$ [21].

In the United States, healthcare providers (HCP) attending pregnant women fall into four main provider types: physicians, such as obstetricians and family practitioners, and midwives including nurse midwives and licensed midwives. Healthcare providers' (HCP) recommendations are given considerable weight by patients, and a pregnant woman who is offered an influenza vaccine by her healthcare provider is more likely to be vaccinated $[11,22]$, but no research has been done on patient compliance with vaccine recommendations 
TABLE 1: Vaccination practices of prenatal care providers.

\begin{tabular}{|c|c|c|c|c|c|}
\hline & $\begin{array}{c}\text { All MDs }(N=81) \\
n(\%)\end{array}$ & $\begin{array}{c}\text { OBs }(N=61) \\
n(\%)\end{array}$ & $\begin{array}{c}\text { FPs }(N=19) \\
n(\%)\end{array}$ & $\begin{array}{c}\text { LMs }(N=25) \\
n(\%)\end{array}$ & $P$ value ${ }^{*}$ \\
\hline \multicolumn{6}{|l|}{ Influenza vaccine } \\
\hline Ask if pregnant patient is up to date & $78(96)$ & $59(97)$ & $18(95)$ & $14(56)$ & $<0.001$ \\
\hline Recommend in pregnancy & $81(100)$ & $61(100)$ & $19(100)$ & $5(20)$ & $<0.001$ \\
\hline Recommend postpartum & $74(91)$ & $56(92)$ & $17(89)$ & $3(12)$ & $<0.001$ \\
\hline \multicolumn{6}{|l|}{ Pertussis vaccine } \\
\hline Ask if pregnant patient is up to date & $68(84)$ & $51(84)$ & $16(84)$ & $10(40)$ & $<0.001$ \\
\hline Recommend in pregnancy & $70(86)$ & $53(87)$ & $17(89)$ & $6(24)$ & $<0.001$ \\
\hline Recommend postpartum & $75(93)$ & $57(93)$ & $17(89)$ & $9(36)$ & $<0.001$ \\
\hline \multicolumn{6}{|l|}{ Will follow ACIP recommendation } \\
\hline Yes/already do & $79(98)$ & $59(97)$ & $19(100)$ & $7(28)$ & $<0.001$ \\
\hline No & $0(0)$ & $0(0)$ & $0(0)$ & $4(16)$ & $<0.001$ \\
\hline Maybe & $2(2)$ & $2(3)$ & $0(0)$ & $14(56)$ & $<0.001$ \\
\hline \multicolumn{6}{|c|}{ Received influenza vaccine in most recent flu season } \\
\hline Yes & $80(99)$ & $60(98)$ & $19(100)$ & $5(25)$ & $<0.001$ \\
\hline
\end{tabular}

${ }^{*} P$ value is chi-square for differences.

based on the type of prenatal provider. We hypothesized that some types of prenatal care providers may immunize at a higher rate than others. Hence, the objectives of this email survey were to compare prenatal care providers' attitudes and practices on influenza and Tdap vaccination in pregnant women.

\section{Materials and Methods}

An email survey was created by staff at the Washington State Department of Health (DOH) and the University of Washington, Department of Obstetrics and Gynecology (UWDOG), using Opinio (Opinio, version 6, Object Planet, Oslo, Norway). Expert survey writers within $\mathrm{DOH}$ reviewed and edited the survey. The survey was pilot tested with obstetricians from the UWDOG.

Potential subjects for the survey were identified from membership lists of the Washington Chapter of the American College of Obstetrics and Gynecology (WA-ACOG) and Washington Obstetrics Association (WOA) and licensing records from the Washington State Department of Licensing (WA-DOL). Overall, 2822 potential respondents were identified in membership and licensure lists: 438 obstetrician (OB) members of WA-ACOG; 154 family practice (FP) physician and midwife members of WOA; 107 licensed midwives (LM) in the WA-DOL records; and 2123 advanced registered nurse practitioners (ARNP) in the WA-DOL records. WA-DOL records do not indicate which ARNPs provide prenatal care. Email addresses were missing for a large proportion of the LMs and ARNPs licensed through the Washington State Department of Health (68\% and $77 \%$ missing, resp.). Because the absolute number of missing emails for LMs was small $(n=73)$, missing emails were searched via the internet and a majority were identified. Due to limited resources, we were unable to search for the missing ARNP emails and so ARNPs, and thus nurse midwives (NM), were excluded from the sample population.

In December 2011, the surveys were successfully emailed to 644 persons: 433 OBs from WA-ACOG list, 120 FPs and midwives from WOA list, and 91 LMs from WA-DOL licensing list. Three email reminders were sent out in the two weeks the survey was open to receive responses. Respondents submitted survey responses online. Inclusion criteria required that the respondent provided prenatal care and was not an NM. The survey was deemed "program evaluation" by Washington Institutional Review Board so was exempt from review. Analysis was performed using Stata 11.0, with chisquare statistic used for comparisons, and Fisher's exact test for cell size $<5$.

\section{Results}

Responses were received from 121 (19\%) of 644 potential respondents, of whom 110 provided prenatal care. Four respondents were NMs and were excluded from analysis, resulting in a final sample size of 106.

Of 106 respondents, 73 (69\%) were female, 61 (58\%) completed an obstetrics and gynecology residency, 19 (18\%) completed a family practice residency, $1(<1 \%)$ had " 50 -year experience" in medical practice, and 25 (24\%) were LMs. By practice type, 81 (76\%) were physicians (MDs) and 25 (24\%) were LMs. Sixty-nine (85\%) of 81 MDs had been licensed for $>10$ years compared to 8 of 25 (32\%) LMs $(P<0.001)$. Thirtyseven $(61 \%)$ of 61 OBs provided care to 20 or more pregnant women per week compared to $3(16 \%)$ of 19 of FPs and $8(32 \%)$ of 25 of LMs $(P<0.001)$.

MDs were more likely to report asking whether pregnant patients were up to date on influenza and Tdap vaccines and to recommend both influenza vaccine and Tdap to pregnant and postpartum women than were LMs $(P<0.001)$ (Table 1$)$. MDs were more likely than LMs to report following the new 
TABLE 2: Capability of prenatal care practices to administer vaccines.

\begin{tabular}{|c|c|c|c|c|c|}
\hline & $\begin{array}{c}\text { All MDs }(N=81) \\
n(\%)\end{array}$ & $\begin{array}{c}\text { OBs }(N=61) \\
n(\%)\end{array}$ & $\begin{array}{c}\text { FPs }(N=19) \\
n(\%)\end{array}$ & $\begin{array}{c}\text { LMs }(N=25) \\
n(\%)\end{array}$ & $P$ value* \\
\hline \multicolumn{6}{|c|}{ Practice administers any vaccines } \\
\hline Yes & $76(94)$ & $56(92)$ & $19(100)$ & $6(24)$ & $<0.001$ \\
\hline \multicolumn{6}{|c|}{ Practice administers specific vaccine } \\
\hline Influenza & $74(97)$ & $54(96)$ & $19(100)$ & $1(17)$ & $<0.001$ \\
\hline Tdap & $66(87)$ & $47(84)$ & $18(95)$ & $3(50)$ & 0.02 \\
\hline \multicolumn{6}{|c|}{ Practice administers vaccines to partners } \\
\hline Yes & $35(46)$ & $18(32)$ & $16(84)$ & $3(50)$ & 0.001 \\
\hline
\end{tabular}

${ }^{*} P$ value is chi-square for differences.

ACIP recommendation for Tdap during pregnancy $(P<$ $0.001)$. MDs were more likely to report having been immunized against influenza in the most recent influenza season than were LMs $(P<0.001)$.

Seventy-six (94\%) of $81 \mathrm{MD}$ practices administered some vaccines to patients compared to only 6 (24\%) of 25 LM practices $(P<0.001)$ (Table 2$)$. Of the $82 \mathrm{MD}$ and LM practices that administered any vaccines, $74(97 \%)$ of $76 \mathrm{MD}$ practices offered influenza vaccine and 66 (87\%) offered Tdap, compared to only $1(17 \%)$ of 6 LM practices that offered influenza vaccine and $3(50 \%)$ that offered Tdap. FP practices were more likely to report administering vaccines to partners of pregnant women than were OB or LM practices $(P=0.001)$.

Of the 74 practices that employed staff, 53 (80\%) of 66 MD practices reported requiring office staff to be vaccinated against influenza compared to 0 of $8 \mathrm{LM}$ practices that employed staff $(P<0.001)$. Thirty $(45 \%)$ MD practices reported requiring office staff to be vaccinated against Tdap compared to 0 of $8 \mathrm{LM}$ practices $(P=0.013)$. Of providers who did not administer vaccines to their patients, reasons given were varied, with the most common being, "Vaccine is out of scope of practice" (Table 3).

\section{Discussion}

A single vaccination of a pregnant woman can provide immune protection to two people: mother and infant. However, despite the focused attention on influenza during the 2009 H1N1 pandemic, with several studies that showed a benefit to infants and mothers from the influenza vaccine, a significant proportion of pregnant women and their infants remain unprotected. In 2003, only $36 \%$ of OBs offered influenza vaccine to pregnant women in their office [23]. A new recommendation by ACIP for Tdap with each pregnancy was announced in 2012. Prenatal care providers are an important and trusted source for medical information to pregnant women [11, 22], and the frequent scheduled visits over the course of a pregnancy provide an opportunity for education and interventions to optimize the health of the mother and infant. This email survey of Washington prenatal care providers suggests that physician prenatal care providers are more likely to report recommending, providing, and receiving influenza vaccine than are LM providers. The findings were similar though less pronounced for Tdap. MDs in this study reported having a high rate of immunization against
TABLE 3: Provider reasons for not giving vaccine.

\begin{tabular}{lc}
\hline Reason & $\begin{array}{c}N=17^{1} \\
n(\%)^{2}\end{array}$ \\
\hline Vaccine is out of scope of practice & $5(29)$ \\
Insurance does not pay & $4(24)$ \\
Do not know how to bill & $2(12)$ \\
Do not have enough staff & $2(12)$ \\
Concerns about vaccine safety & $2(12)$ \\
Too busy & $1(6)$ \\
Do not know how to order vaccine & $1(6)$ \\
Do not know how to set up vaccination program & 0 \\
Concerns about liability & 0 \\
Do not think vaccines necessary & 0
\end{tabular}

${ }^{1}$ Of respondents, 17 did not offer one or both vaccines.

${ }^{2}$ Multiple responses were allowed.

influenza and of offering vaccines in the office. FP practices appear more able than OB or LM practices to provide vaccines to partners of pregnant women.

This study had several limitations. Results may not be generalizable for several reasons; lists of email addresses were incomplete, unequal searches for email addresses may have introduced some bias, and the response rate was low. As with any survey, answers provided by responders may not be completely representative of nonresponders. Regional differences in vaccination practices may be present in the United States and this study only addressed practices in a single state. Adequate data do not exist defining the proportion of MD and different types of midwives providing prenatal care, so we cannot determine whether the proportions in the study population are representative of provider types attending pregnant women in Washington. Data from the Centers for Disease Control and Prevention suggest that approximately $7.6 \%$ of births in the US were attended by nurse midwives [24] but do not address LMs who tend to have fewer training requirements. Given that only about half of all pregnant women receive an influenza vaccine, whereas virtually all MD survey respondents were strong proponents of influenza vaccine for pregnant women, our results suggest that the MD study population may not be representative of all MDs providing prenatal care. However, these results suggest that differences may exist in promotion of vaccines by different types of prenatal 
care providers and that LMs may be less likely to recommend vaccines to pregnant women. This preliminary study does not adequately sample all provider populations nor differentially assess patient feelings about vaccine based on provider type; however, the difference in provider attitudes toward vaccination deserves further study in a larger sample.

Solid evidence exists that influenza vaccination of pregnant women can prevent complications of influenza in mothers and infants and a focus on timely vaccination of pregnant women is recommended. Uptake of the pertussis vaccine by pregnant women since the most recent ACIP recommendation remains to be determined. Optimizing adherence to the standard of care recommendations for pregnant women will improve outcomes for a healthy start in life for infants. The results of our study provide a starting point for educational communication with the LM community to assess the validity of our results and to identify opportunities for improving education about current vaccine recommendations for pregnant women.

\section{Conflict of Interests}

The authors declare that there is no conflict of interests regarding the publication of this paper.

\section{References}

[1] J. K. Louie, M. Acosta, D. J. Jamieson, and M. A. Honein, "Severe 2009 H1N1 influenza in pregnant and postpartum women in California," The New England Journal of Medicine, vol. 362, no. 1, pp. 27-35, 2010.

[2] D. J. Jamieson, M. A. Honein, S. A. Rasmussen et al., "H1N1 2009 influenza virus infection during pregnancy in the USA," The Lancet, vol. 374, no. 9688, pp. 451-458, 2009.

[3] T. V. Hartert, K. M. Neuzil, A. K. Shintani et al., "Maternal morbidity and perinatal outcomes among pregnant women with respiratory hospitalizations during influenza season," The American Journal of Obstetrics and Gynecology, vol. 189, no. 6, pp. 1705-1712, 2003.

[4] Centers for Disease Control and Prevention, "Maternal and infant outcomes among severely ill pregnant and postpartum women with 2009 pandemic influenza A (H1N1)_United States, April 2009-August 2010," Morbidity and Mortality Weekly Report, vol. 60, no. 35, pp. 1193-1196, 2011.

[5] A. A. Eick, T. M. Uyeki, A. Klimov et al., "Maternal influenza vaccination and effect on influenza virus infection in young infants," Archives of Pediatrics and Adolescent Medicine, vol. 165, no. 2, pp. 104-111, 2011.

[6] I. Benowitz, D. B. Esposito, K. D. Gracey, E. D. Shapiro, and M. Vázquez, "Influenza vaccine given to pregnant women reduces hospitalization due to influenza in their infants," Clinical Infectious Diseases, vol. 51, no. 12, pp. 1355-1361, 2010.

[7] M. C. Steinhoff, S. B. Omer, E. Roy et al., "Influenza immunization in pregnancy-antibody responses in mothers and infants," The New England Journal of Medicine, vol. 362, no. 17, pp. 16441646, 2010.

[8] K. Zaman, E. Roy, S. E. Arifeen et al., "Effectiveness of maternal influenza immunization in mothers and infants," The New England Journal of Medicine, vol. 359, no. 15, pp. 1555-1564, 2008.
[9] Centers for Disease Control and Prevention, "Prevention and control of influenza: recommendations of the advisory committee on immunization practices (ACIP)," Morbidity and Mortality Weekly Report, vol. 46, no. 9, pp. 1-24, 1997.

[10] Centers for Disease Control and Prevention, "Prevention and control of influenza: recommendations of the advisory committee on immunization practices (ACIP)," Morbidity and Mortality Weekly Report, vol. 53, no. 6, pp. 1-40, 2004.

[11] Centers for Disease Control and Prevention, "Influenza vaccination coverage among pregnant women-29 States and New York City, 2009-2010 Season," Morbidity and Mortality Weekly Report, vol. 61, no. 07, pp. 113-118, 2012.

[12] C. R. Vitek, F. B. Pascual, A. L. Baughman, and T. V. Murphy, "Increase in deaths from pertussis among young infants in the United States in the 1990s," Pediatric Infectious Disease Journal, vol. 22, no. 7, pp. 628-634, 2003.

[13] N. Wortis, P. M. Strebel, M. Wharton, B. Bardenheier, and I. R. B. Hardy, "Pertussis deaths: report of 23 cases in the United States, 1992 and 1993," Pediatrics, vol. 97, no. 5, pp. 607-612, 1996.

[14] A. M. Wendelboe, E. Njamkepo, A. Bourillon et al., "Transmission of Bordetella pertussis to young infants," Pediatric Infectious Disease Journal, vol. 26, no. 4, pp. 293-299, 2007.

[15] K. M. Bisgard, F. B. Pascual, K. R. Ehresmann et al., "Infant pertussis: who was the source?" Pediatric Infectious Disease Journal, vol. 23, no. 11, pp. 985-989, 2004.

[16] C. M. Healy, F. M. Munoz, M. A. Rench, N. B. Halasa, K. M. Edwards, and C. J. Baker, "Prevalence of pertussis antibodies in maternal delivery, cord, and infant serum," Journal of Infectious Diseases, vol. 190, no. 2, pp. 335-340, 2004.

[17] S. A. Gall, J. Myers, and M. Pichichero, "Maternal immunization with tetanusdiphtheriapertussis vaccine: effect on maternal and neonatal serum antibody levels," The American Journal of Obstetrics and Gynecology, vol. 204, no. 4, pp. 334.el-334.e5, 2011.

[18] J. H. Shakib, S. Ralston, H. H. Raissy, G. J. Stoddard, K. M. Edwards, and C. L. Byington, "Pertussis antibodies in postpartum women and their newborns," Journal of Perinatology, vol. 30, no. 2, pp. 93-97, 2010.

[19] E. Leuridan, N. Hens, N. Peeters, L. de Witte, O. van der Meeren, and P. van Damme, "Effect of a prepregnancy pertussis booster dose on maternal antibody titers in young infants," Pediatric Infectious Disease Journal, vol. 30, no. 7, pp. 608-610, 2011.

[20] CDC, "Updated recommendations for use of tetanus toxoid, reduced diphtheria, and acellular pertussis vaccine (Tdap) in pregnant women-advisory committee on immunization practices (ACIP), 2012," Morbidity and Mortality Weekly Report, vol. 62, no. 7, pp. 131-135, 2013.

[21] Centers for Disease Control and Prevention, "Adult vaccination coverage-United States, 2010," Morbidity and Mortality Weekly Report, vol. 61, no. 4, pp. 66-72, 2012.

[22] Centers for Disease Control and Prevention, "Influenza vaccination coverage among pregnant women-United States, 201011 influenza season," Morbidity and Mortality Weekly Report, vol. 60, no. 32, pp. 1078-1082, 2011.

[23] Centers for Disease Control and Prevention, "Influenza vaccination in pregnancy: practice among obstetrician-gynecologists-United States, 2003-04 influenza season," Morbidity and Mortality Weekly Report, vol. 54, no. 41, pp. 1050-1052, 2005.

[24] E. Declercq, "Trends in midwife-attended births in the United States, 1989-2009," Journal of Midwifery \& Women's Health, vol. 57, no. 4, pp. 321-326, 2012. 


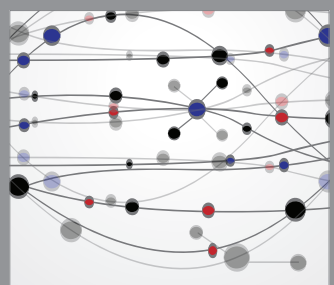

The Scientific World Journal
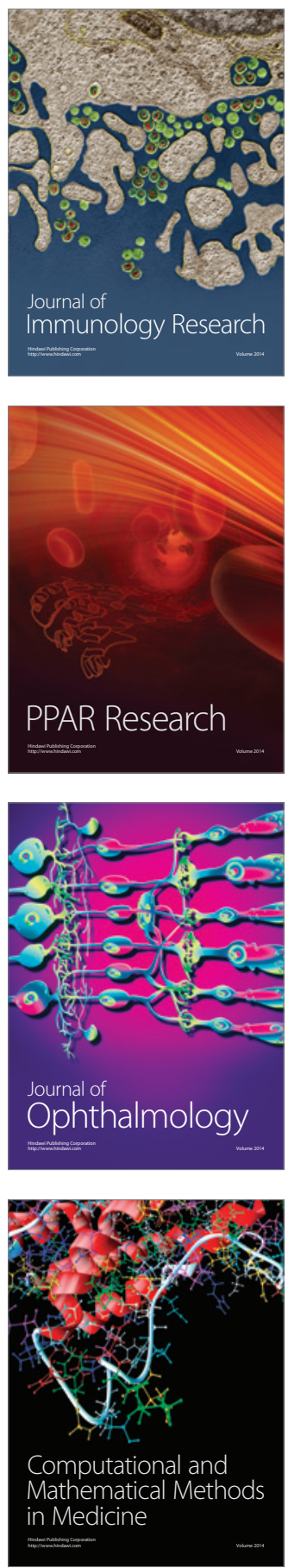

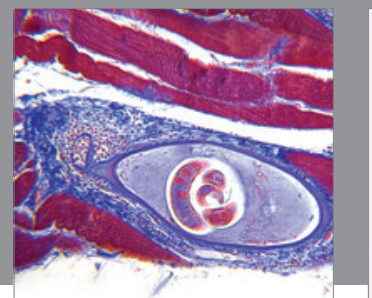

Gastroenterology

Research and Practice
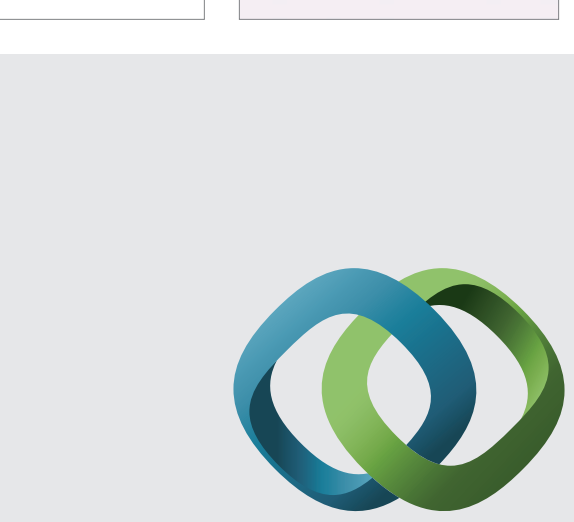

\section{Hindawi}

Submit your manuscripts at

http://www.hindawi.com
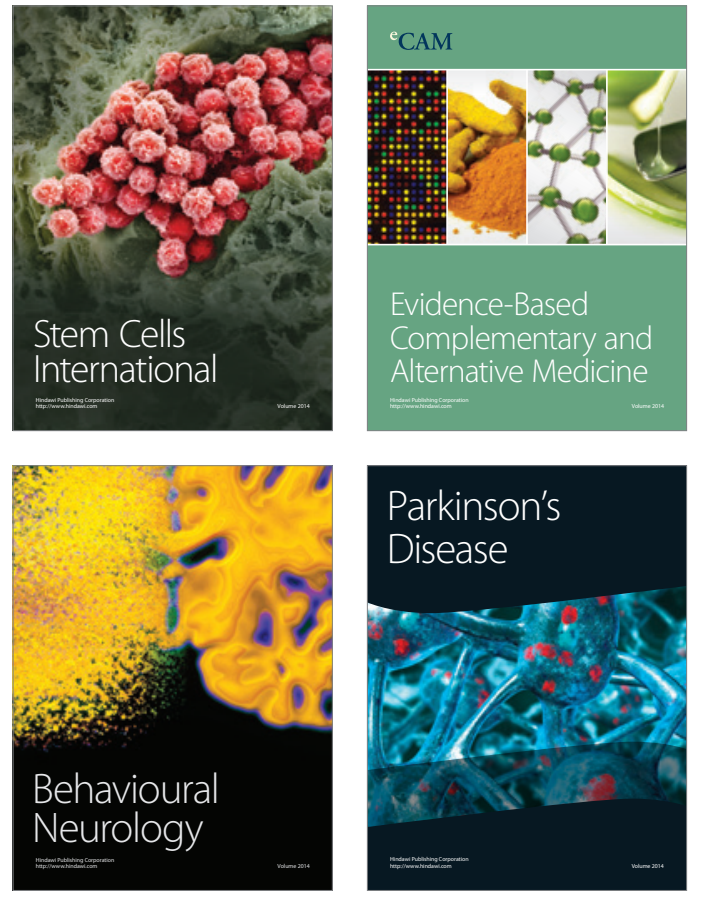
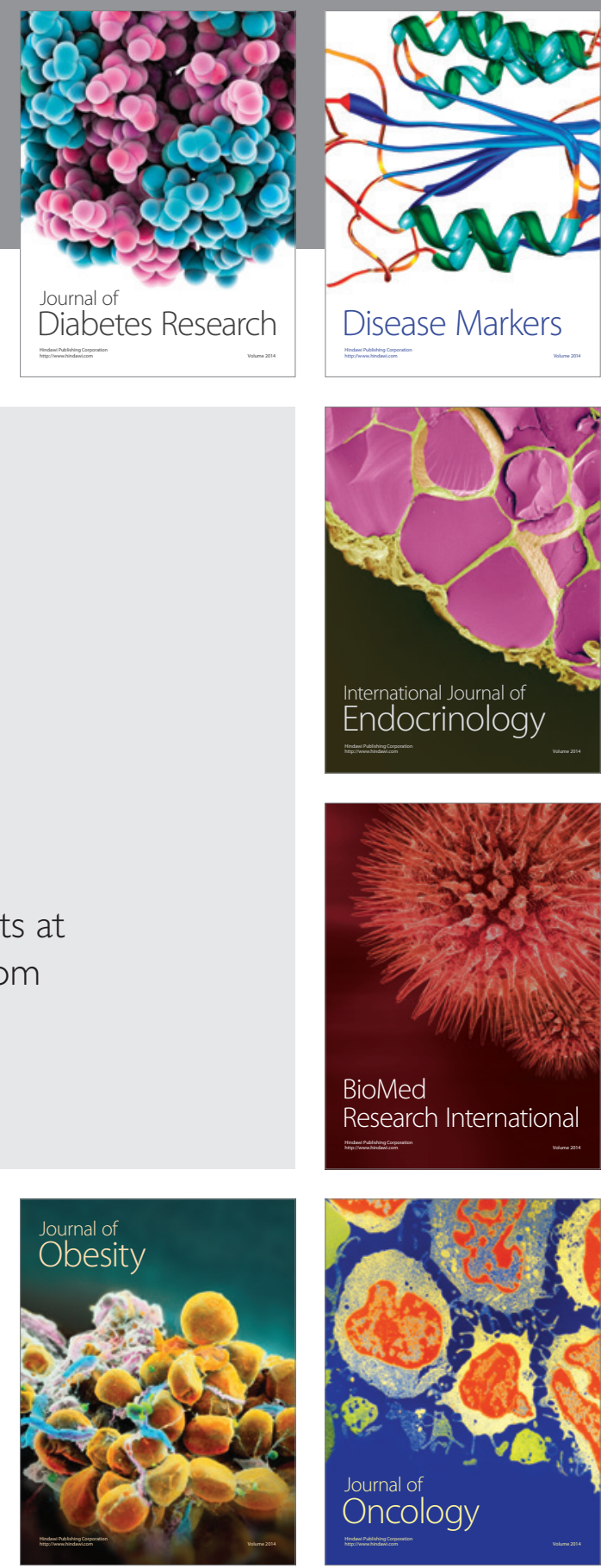

Disease Markers
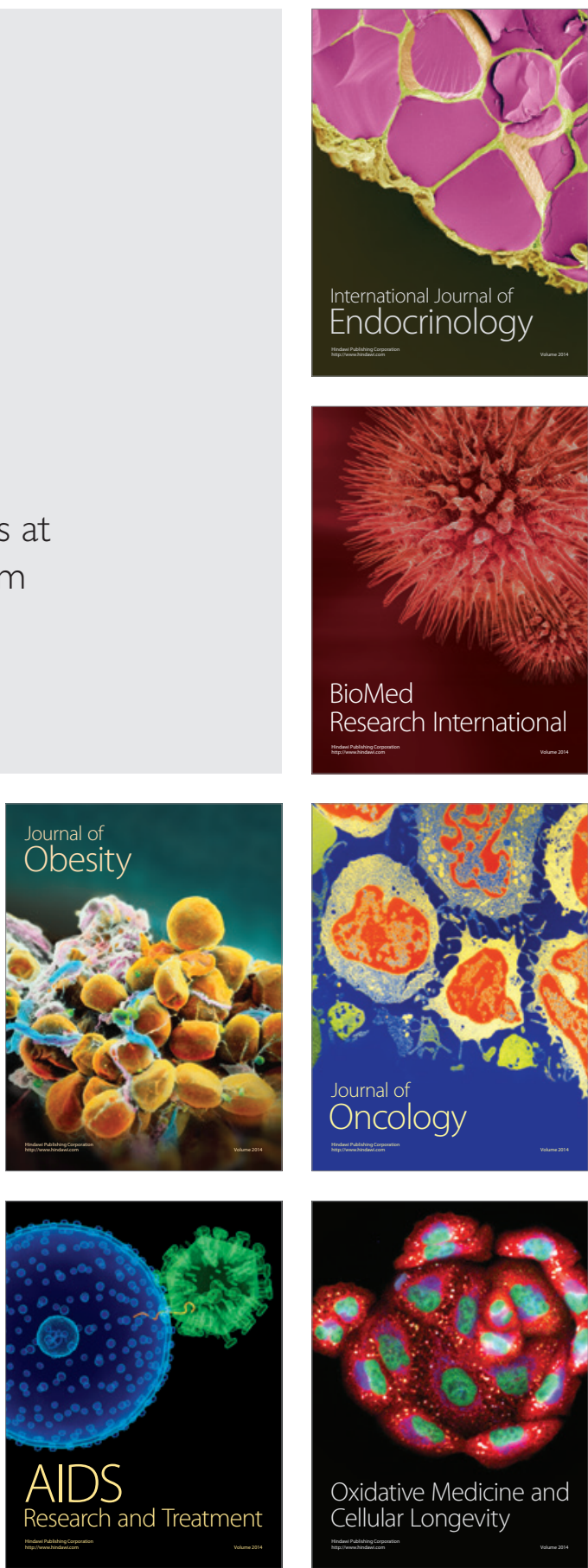